Article

\title{
Introduction to Dependence Relations and Their Links to Algebraic Hyperstructures
}

\author{
Irina Cristea ${ }^{1, * \mathbb{D}}$, Juš Kocijan ${ }^{1,2} \mathbb{D}$ and Michal Novák ${ }^{3} \mathbb{D}$ \\ 1 Centre for Information Technologies and Applied Mathematics, University of Nova Gorica, \\ 5000 Nova Gorica, Slovenia; jus.kocijan@ijs.si \\ 2 Department of Systems and Control, Jožef Stefan Institut, Jamova Cesta 39, 1000 Ljubljana, Slovenia \\ 3 Faculty of Electrical Engineering and Communication, Brno University of Technology, Technická 10, \\ 61600 Brno, Czech Republic; novakm@feec.vutbr.cz \\ * Correspondence: irina.cristea@ung.si or irinacri@yahoo.co.uk; Tel.: +386-533-153-95
}

Received: 11 July 2019; Accepted: 19 September 2019; Published: 23 September 2019

\begin{abstract}
The aim of this paper is to study, from an algebraic point of view, the properties of interdependencies between sets of elements (i.e., pieces of secrets, atmospheric variables, etc.) that appear in various natural models, by using the algebraic hyperstructure theory. Starting from specific examples, we first define the relation of dependence and study its properties, and then, we construct various hyperoperations based on this relation. We prove that two of the associated hypergroupoids are $H_{v}$-groups, while the other two are, in some particular cases, only partial hypergroupoids. Besides, the extensivity and idempotence property are studied and related to the cyclicity. The second goal of our paper is to provide a new interpretation of the dependence relation by using elements of the theory of algebraic hyperstructures.
\end{abstract}

Keywords: hyperoperation; hypergroupoid; dependence relation; influence; impact

\section{Introduction}

In many real-life situations, there are contexts with numerous "variables", which somehow depend on one another. Consider, for instance, a group of people sharing several secrets, which are not known to everybody because some subgroups, which overlap arbitrarily, share only some secrets; or we have several measurement stations, such as those used in weather forecasting, which measure some data used for predictions for some other places in some future time. In this context, all we know is that the prediction will somehow depend on some of the measured data, but we do not know how or exactly on which specific data. This is the same as revealing one secret to one person. Again, we know that this will have an influence on the overall level of secrecy in our group of people, but it is rather difficult to tell who will find out what, for the simple reason that neither people nor Nature act like machines. Alternatively, we can consider a relational database with many relations between entries, in which updating or deleting an entry will have an influence on the overall validity of the database. Therefore, a mathematical tool is needed to describe and model such contexts. The notion of "functional dependence" was used to describe this in the theory of database relations as early as the 1970s; see, e.g., Armstrong [1] or the references of Matúš [2]. In the context of secrets, see, e.g., More and Naumov [3] and their references.

Now, we will show the precise way of describing this concept mathematically using functional dependence relations. For an arbitrary finite set $H$ and arbitrary nonempty subsets $A, B, C \subseteq H$, we read $A \triangleright B$ as " $B$ depends functionally on $A$ ". This relation is considered reflexive, i.e., if $A \supseteq B$, then $A \triangleright B$, transitive, i.e., if $A \triangleright B$ and $B \triangleright C$, then $A \triangleright C$, and an axiom of augmentation is defined for it, which means that if $A \triangleright B$, then also $A \cup C \triangleright B \cup C$. In the above-mentioned context of secrets, " $a \triangleright b$ " 
reads as "the value of secret $b$ depends on the value of secret $a$ ", which is equivalent to the fact that the value of secret $a$ reveals the value of secret $b$ or to the fact that the value of secret $a$ influences the value of secret $b$. In the context of sets, we can extend the relation to sets writing $A \triangleright B$ for the fact that the values of all secrets in the set $A$ reveal the values of all secrets in the set $B$.

The aim of this paper is to study, from an algebraic point of view, the properties of inter-dependencies between sets of elements (i.e., pieces of secrets, atmospheric variables, etc.) that appear in various natural models, by using the algebraic hyperstructure theory. Starting from specific examples, we first define the relation of dependence, study its properties, and then, we construct various hyperoperations based on this relation. The second goal of our paper is to provide a new interpretation of the dependence relation by using elements of the theory of algebraic hyperstructures.

The rest of the paper is organized as follows: In Section 2, the motivation of this study is described, based on a concrete example related to the measurement of a set of atmospheric variables. Section 3 gathers the definition and main algebraic properties of the new dependence relations that describe the behavior of the variables modeled in the previous section. An overview of the theory of algebraic hyperstructures is presented in Section 4, while some applications of the dependence relations to the theory of hyperstructures are proposed in Section 5. The paper ends with some concluding ideas and future work related to a numerical interpretation of these dependencies.

\section{Motivation}

Our paper is motivated not by the context of secrets or database relations, but by the second above-mentioned context, i.e., weather forecasting using data measured at various measurements stations. These variables are treated as time-dependent signals, which describe the direction and speed of wind in the key point of the atmosphere, the turbulent nature of the atmosphere, thermal stratification, and the local non-homogeneity of particular variables, as well as many other variables. Existing weather forecasts can only approximate these values even if they are made with a dedicated modeling system specially tailored for forecasts in fine spatial and temporal resolution over complex terrain. Obviously, the measured values are interrelated in some ways, some of which can be multiple, can overlap, depend on one another, etc. Therefore, our first aim is to define a new type of dependence relation in order to model this context. Then, given the nature of the context, we are going to associate some algebraic hyperstructures with them and study their properties with the final goal to better understand the newly-defined dependence relations.

As regards the weather forecasting context, we will treat a specific situation [4,5]. There are four measurement stations located near Krško nuclear power plant in Slovenia ("Krško NPP"). Out of these, we get a set of variables describing temperature, wind, humidity, etc., that are also measured in Krško NPP. Many dependencies can be described, and many of these are redundant or are linked in a certain way. In order to make the prediction model as simple, accurate, and fast as possible, we need to find out a "minimal set" of these dependencies that describe as many aspects of the system as possible.

In the existing research [5], the measured data have the form of atmospheric variables originating from different measurement stations (Brežice measurement station (B), Cerklje measurement station (C), Cerklje airport measurement station (CA), Krško measurement station (K), Stolp Krško measurement station at Krško NPP (S)): temperature $2 \mathrm{~m}$ above the ground (1), wind speed $10 \mathrm{~m}$ above the ground (2), wind direction $10 \mathrm{~m}$ above the ground (3), relative humidity $2 \mathrm{~m}$ above the ground (4), air pressure (5), temperature $10 \mathrm{~m}$ above the ground (6), temperature $40 \mathrm{~m}$ above the ground (7), temperature $70 \mathrm{~m}$ above the ground (8), and global solar radiation (9). Besides measurements at measurement stations, we have available also forecasts from a physics-based model, hereafter called the WRF model [5], that does not correspond exactly to the Krško NPP location. The existing research [5] shows that there are some links between the values of some of these variables as follows.

The variable representing temperature $2 \mathrm{~m}$ above the ground at Stolp Krško measurement station in the moment $k$, denoted $S_{k, 1}$, depends with the nonlinear functional dependence on other variables as shown in Table 1. 
Table 1. The nonlinear functional dependence of variable $S_{k, 1}$.

\begin{tabular}{llll}
\hline Measurement Station & Variable & The Time of Sample & Notation \\
\hline Stolp Krško & temperature at 2 m & $k-2$ & $S_{k-2,1}$ \\
Stolp Krško & temperature at 2 m & $k-1$ & $S_{k-1,1}$ \\
Stolp Krško & global solar radiation & $k-2$ & $S_{k-2,9}$ \\
Cerklje & temperature at 2 m & $k-1$ & $C_{k-1,1}$ \\
Stolp Krško & wind speed & $k-1$ & $S_{k-1,2}$ \\
Stolp Krško & global solar radiation & $k-1$ & $S_{k-1,9}$ \\
WRF model & temperature at 2 m & $k$ & $W_{k, 1}$ \\
WRF model & global solar radiation & $k$ & $W_{k, 9}$ \\
\hline
\end{tabular}

This can be written as $S_{k, 1} \sim D\left(S_{k-2,1}, S_{k-1,1}, S_{k-2,9}, C_{k-1,1}, S_{k-1,2}, S_{k-1,9}, W_{k, 1}, W_{k, 9}\right)$. Similarly, other dependencies exist, and we write all of them as follows (they will be used later in Example 1):

$$
\begin{aligned}
& S_{k, 1} \sim D\left(S_{k-2,1}, S_{k-1,1}, S_{k-2,9}, C_{k-1,1}, S_{k-1,2}, S_{k-1,9}, W_{k, 1}, W_{k, 9}\right) \\
& S_{k, 6} \sim D\left(S_{k-2,6}, S_{k-1,6}, K_{k-2,1}, C_{k-1,1}, K_{k-1,1}, K_{k-1,2}, W_{k, 1}\right) \\
& S_{k, 7} \sim D\left(S_{k-2,7}, S_{k-1,7}, B_{k-2,4}, C_{k-2,1}, C_{k-2,4}, C A_{k-2,1}, K_{k-2,2}, K_{k-2,3}, S_{k-2,4}, W_{k, 2}, W_{k, 1}\right) \\
& S_{k, 8} \sim D\left(S_{k-1,8}, S_{k-2,8}, C_{k-2,1}, S_{k-1,9}, W_{k, 1}\right) .
\end{aligned}
$$

In the notation of the variables, the time of sample $k-1$ means it was measured half an hour in the past, and the time of sample $k-2$ means an hour in the past from the present time denoted as $k$. All variables' values are measured at consequent and equidistant time instants $k$ multiplied by the value of the sampling time, which in our case is half an hour, and are mutually independent and independent of the values of all other variables.

Since we are interested mainly in developing a new algebra for modeling this context based on the new dependence relations, at this stage of the research, we do not make use of the existing numerically-established links discussed, e.g., in [5]. We intend to propose a new algebraic abstract model for identifying the level of importance of certain variables in the prediction, which will show which variables can be replaced by others, ignored without influencing the prediction above a reasonable limit, etc.

\section{Properties of Dependence Relations}

\subsection{Definition of a Dependence Relation}

Let $H$ be a finite set of variables of cardinality $n$, denoted as $a_{i, j}$, where the double index is motivated by the above example. There exist several links between some of these variables represented as dependencies, which we will denote as follows: For an arbitrary $k+1$-tuple of variables, denote:

$$
a_{i_{0}, j_{0}} \sim D\left(a_{i_{1}, j_{1}}, a_{i_{2}, j_{2}}, \ldots, a_{i_{k}, j_{k}}\right),
$$

which will be a shortcut for the fact that the variable $a_{i_{0}, j_{0}}$ depends on the variables on the right-hand side of the relation, which is equivalent to the fact that the variables on the right-hand side influence the variables on the left-hand side. Notice, that on the left-hand side, we will always, by definition, have one variable only. Furthermore, by saying that some components depend on some other ones, we do not assign any preferences or weights. Therefore, for the time being, the order of elements in the dependency is not relevant. This leads us to the following rather loose and general definition. 
Definition 1. By a dependence relation, we mean a formula between several variables with a left-and right-hand side, expressing that the value of the variable on the left-hand side depends (in an unspecific way) on the values of the variables on the right-hand side.

It is to be pointed out that even though in our considerations, we used the word "relation", we do not use it in the established algebraic sense of the $n$-ary relation with fixed $n$ and standard properties such as reflexivity, transitivity, etc.

Indeed, consider for example $x \sim D(y, z, t)$, which can be written as $(x, y, z, t) \in D$ (however, with $x$ always in the first position), suggesting that we work with a relation of arity four. In this case, reflexivity means that for every $x$ in the considered set (called support set), there is $(x, x, x, x) \in D$, which in our case would be written as $x \sim D(x, x, x)$, which in fact has no sense because we always have only $x \sim D(x)$, i.e., $(x, x) \in D$. Yet, obviously, in real life, such relations are always reflexive, i.e., $x \sim D(x)$ for all $x \in H$. However, since this is a trivial assumption, we will not mention this in a list of dependencies. If, for a certain element, there exists only the dependence $x \sim D(x)$, we call $x$ an isolated element.

Furthermore, rather than the transitivity of our relations, we should consider their compositions of a specific type. Consider, e.g., three dependencies:

$$
\begin{aligned}
a_{1,1} & \sim D\left(a_{1,2}, a_{2,2}, a_{3,2}, a_{3,5}\right) \\
a_{2,2} & \sim D\left(a_{2,3}, a_{3,1}\right) \\
a_{3,2} & \sim D\left(a_{1,2}, a_{3,4}\right)
\end{aligned}
$$

which can be written as:

$$
a_{1,1} \sim D\left(a_{1,2}, a_{2,2} \sim D\left(a_{2,3}, a_{3,1}\right), a_{3,2} \sim D\left(a_{1,2}, a_{3,4}\right), a_{3,5}\right) .
$$

Notice that, in the general case, the relation " $\sim$ " is not symmetric because-unless stated explicitly by another dependency - e.g., the value of $a_{1,1}$ depends on $a_{1,2}$, but not vice versa.

Furthermore, we point out that our usage of the language of functional dependencies is rather loose and only freely inspired by the axiomatic system of Armstrong [1], which is used in database theory, secrets modeling [3], etc. This is because our motivational example of weather forecasting has some properties that cannot be assumed under Armstrong's system. For example, transitivity or augmentation axioms will make no sense in our context (or would be trivial). Indeed, as far as augmentation is concerned, regard, e.g., relation $a_{1,2} \sim D\left(a_{2,3}, a_{3,4}\right)$. This would mean, for example, that $a_{1,2} \cup a_{1,1} \sim D\left(a_{2,3}, a_{3,4}, a_{1,1}\right)$, i.e., $a_{1,1}$ depends somehow on $a_{2,3}$ and $a_{3,4}$, which need not be true.

\subsection{Influence and Impact in a Dependence Relation}

If we have the dependence $a_{1,1} \sim D\left(a_{1,2}, a_{1,3}, a_{2,3}, a_{2,1}\right)$, we read it as " $a_{1,1}$ depends on $a_{1,2}, a_{1,3}, a_{2,3}, a_{2,1}$ " without any order preferences. Such a statement is obviously equivalent to saying " $\left\{a_{1,2}, a_{1,3}, a_{2,3}, a_{2,1}\right\}$ is the set of components of the dependence that has a certain influence on the element $a_{1,1}$ ". Besides, we always consider that an element has a certain influence on itself, even though this is not clearly evident in the written form of the dependence, so we will avoid writing $a_{1,1} \sim D\left(a_{1,1}, a_{1,2}, a_{1,3}, a_{2,3}, a_{2,1}\right)$. Thus, in this respect, we will speak of influential elements of $a_{1,1}$ and write $\operatorname{Infl}\left(a_{1,1}\right)=\left\{a_{1,1}, a_{1,2}, a_{1,3}, a_{2,3}, a_{2,1}\right\}$. Notice that the influential elements appear on the right-hand side of the relation. Elements that do not appear on the right-hand side of any of the dependencies, but appear on the left-hand side of at least one dependence, will be called non-influential elements.

Now, consider two dependencies: $a_{1,1} \sim D\left(a_{1,2}, a_{1,3}, a_{2,3}, a_{2,1}\right)$ and $a_{2,3} \sim D\left(a_{1,2}, a_{2,2}\right)$. We can see that $a_{1,2}$ is an influential element of both $a_{1,1}$ and $a_{2,3}$ or, in other words, that $a_{1,2}$ has some impact on $a_{1,1}$ and $a_{2,3}$. We can better describe this property by introducing a new set, $\operatorname{Imp}\left(a_{1,2}\right)=\left\{a_{1,1}, a_{2,3}, a_{1,2}\right\}$ 
as the set of all elements on which $a_{1,2}$ has an impact. Thus, such elements will be called elements influenced by $a_{1,2}$. Notice that in the relations, such elements appear on the left-hand side.

In other words, for any element $x$ of the support set, we define two sets:

- $\quad \operatorname{Infl}(x)$ as the set of all influential elements of $x$,

- $\operatorname{Imp}(x)$ as the set of all elements influenced by $x$.

Remark 1. Further on, we will often make use of the obvious fact that, for any two elements of the support set $x, y$, the fact that $y \in \operatorname{Infl}(x)$ is equivalent to the fact that $x \in \operatorname{Imp}(y)$.

Obviously, both of these sets are non-empty because there is always $x \sim D(x)$, i.e., $x \in \operatorname{Infl}(x) \cap$ $\operatorname{Imp}(x)$. With respect to the above-discussed isolated elements, it is obvious that $x$ is isolated if and only if $|\operatorname{Infl}(x)|=1=|\operatorname{Imp}(x)|$, while there is $|\operatorname{Imp}(y)|=1$ and $|\operatorname{Infl}(y)|>1$ for non-influential elements.

\subsection{Simple and Composed Dependencies}

Another important property of the dependence relation is the composition, which helps us distinguish between what is intuitively a "simple" dependence and a "composed" one. Consider again the three dependencies of (6). Instead of:

$$
a_{1,1} \sim D\left(a_{1,2}, a_{2,2} \sim D\left(a_{2,3}, a_{3,1}\right), a_{3,2} \sim D\left(a_{1,2}, a_{3,4}\right), a_{3,5}\right)
$$

we can also write:

$$
a_{1,1} \sim D\left(a_{1,2}, D\left(a_{2,3}, a_{3,1}\right), D\left(a_{1,2}, a_{3,4}\right), a_{3,5}\right) .
$$

Using the former notation, no information on influential elements is lost, i.e., it still can be seen that $a_{2,2}, a_{3,2} \in \operatorname{Infl}\left(a_{1,1}\right)$. However, this information is lost in the latter notation. Anyway, we can call the above dependence (no matter which notation is used) composed because the symbol $D$ appears at least two times in the notation. On the other hand, if it appeared only once, as is the case of, e.g., $a_{3,2} \sim D\left(a_{1,2}, a_{3,4}\right)$, we would call the dependence simple. We can conclude that the composed dependencies are obtained from simple ones using composition.

What else can we say about composed dependencies? Let us consider (6) again. We have:

$$
a_{1,1} \sim D\left(a_{1,2}, a_{2,2} \sim D\left(a_{2,3}, a_{3,1}\right), a_{3,2} \sim D\left(a_{1,2}, a_{3,4}\right), a_{3,5}\right)
$$

and we get that:

$$
\operatorname{Infl}\left(a_{1,1}\right)=\left\{a_{1,1}, a_{1,2}, a_{2,2}, a_{3,2}, a_{3,4}\right\} \cup\left\{a_{2,3}, a_{3,1}, a_{1,2}, a_{3,4}\right\}=\operatorname{Infl}_{d}\left(a_{1,1}\right) \cup \operatorname{Infl}_{i}\left(a_{1,1}\right),
$$

where $\operatorname{Infl}_{d}\left(a_{1,1}\right)$ contains the influential elements of $a_{1,1}$, which appear in a simple dependence of $a_{1,1}$. For this reason, we shall call them direct influential elements. The second set of $(8), \operatorname{Infl}_{i}\left(a_{1,1}\right)$, has a somewhat "indirect" impact on $a_{1,1}$ since its elements appear in simple dependencies of $a_{2,2}$ and $a_{3,2}$, which are nested in the main considered dependence. This makes us call them indirect influential elements of $a_{1,1}$. Notice that the notion of direct or indirect influence is always linked to a given element, i.e., one element may be simultaneously a direct influential element (of $x$ ), as well as an indirect influential element (of $y$ ). Moreover, because there is always $x \sim D(x)$, we include in the set of the direct influential elements of $x$ also $x$ itself, even though the trivial dependence is not important in our considerations, and we call it an improper influential element, while all the other influential elements are called proper.

\subsection{Length and Level of a Dependence Relation}

Let us start with a simple dependence $D$. By the length of $D$, denoted by length $(D)$, we mean the number of the distinct elements that influences a given element in $D$. For example, if there 
is $a \sim D\left(b_{1}, b_{2}, b_{3}\right)$, then length $(D)=3$. Consider now a more complicated case of a composed dependence. Once again, we will use dependence (6), i.e.,

$$
a_{1,1} \sim D\left(a_{1,2}, a_{2,2} \sim D\left(a_{2,3}, a_{3,1}\right), a_{3,2} \sim D\left(a_{1,2}, a_{3,4}\right), a_{3,5}\right)
$$

for which we have seen in (8) that $\operatorname{Infl}\left(a_{1,1}\right)$ consists of two sets. The length of the dependence $D$ is the cardinality of the set of proper influential elements, i.e., length $(D)=\left|\operatorname{Infl}\left(a_{1,1}\right)\right|=7$.

Furthermore, we can say that $a_{1,1}$ appears in a dependence of length seven and Level 2, because the symbol $D$ is nested once (i.e., there is a dependence within a dependence). The level of a simple dependence is always one, while in a general case, the level of a composed dependence is $k$ when $k-1$ dependencies are nested in one another. In a certain way, this concept is similar to the order of a differential equation, which is linked to the highest order of the derivative. For example, the level of the composed dependence $a_{1,1} \sim D\left(a_{1,2}, a_{1,3} \sim D\left(a_{2,3}, a_{3,1} \sim D\left(a_{4,5}, a_{4,1}\right)\right), a_{3,2} \sim D\left(a_{1,2}, a_{3,4}\right), a_{3,5}\right)$ is three.

Example 1. Consider the motivating example of this research concerning the atmospheric variables of the five measurement stations situated near Krško nuclear power plant described in Section 2 and refer to the dependence relations among them, grouped in Label (1). We immediately can say that four distinct simple dependence relations of Level 1 exist, with lengths of 8,7,11, and, respectively, 5, but this is not the perfect description of the real model, since each of the four relations holds for each time instant $k$, so for example, we have also the dependencies:

$$
\begin{aligned}
& S_{k-1,8} \sim D\left(S_{k-2,8}, S_{k-3,8}, C_{k-3,1}, S_{k-2,9}, W_{k-1,1}\right) \\
& S_{k-2,8} \sim D\left(S_{k-3,8}, S_{k-4,8}, C_{k-4,1}, S_{k-3,9}, W_{k-2,1}\right),
\end{aligned}
$$

if we refer only to the last relation in Label (1). In other words, all four dependencies are composed, of superior levels. However, here, we will ignore this property, because it refers only to the time and not to the name of the variable.

Regarding the sets of all influential elements of a certain element, we notice that:

$$
\begin{aligned}
& \operatorname{Infl}\left(S_{k, 1}\right)=\left\{S_{k, 1}, S_{k-2,1}, S_{k-1,1}, S_{k-2,9}, C_{k-1,1}, S_{k-1,2}, S_{k-1,9}, W_{k, 1}, W_{k, 9}\right\} \\
& \operatorname{Infl}\left(S_{k, 6}\right)=\left\{S_{k, 6}, S_{k-2,6}, S_{k-1,6}, K_{k-2,1}, C_{k-1,1}, K_{k-1,1}, K_{k-1,2}, W_{k, 1}\right\} \\
& \operatorname{Infl}\left(S_{k, 7}\right)=\left\{S_{k, 7}, S_{k-2,7}, S_{k-1,7}, B_{k-2,4}, C_{k-2,1}, C_{k-2,4}, C A_{k-2,1}, K_{k-2,2}, K_{k-2,3}, S_{k-2,4}, W_{k, 2}, W_{k, 1}\right\} \\
& \operatorname{Infl}\left(S_{k, 8}\right)=\left\{S_{k, 8}, S_{k-2,8}, S_{k-1,8}, C_{k-2,1}, S_{k-1,9}, W_{k, 1}\right\},
\end{aligned}
$$

while for any element $x \notin\left\{S_{k, 1}, S_{k, 6}, S_{k, 7}, S_{k, 8}\right\}$, there is $\operatorname{Infl}(x)=\{x\}$.

Regarding now the impact sets, those being more significant, containing more than two elements, are:

$$
\begin{aligned}
\operatorname{Imp}\left(C_{k-1,1}\right) & =\left\{C_{k-1,1}, S_{k, 1}, S_{k, 6}\right\} \\
\operatorname{Imp}\left(C_{k-2,1}\right) & =\left\{C_{k-2,1}, S_{k, 7}, S_{k, 8}\right\} \\
\operatorname{Imp}\left(S_{k-1,9}\right) & =\left\{S_{k-1,9}, S_{k, 1}, S_{k, 8}\right\} \\
\operatorname{Imp}\left(W_{k, 1}\right) & =\left\{W_{k, 1}, S_{k, 1}, S_{k, 6}, S_{k, 7}, S_{k, 8}\right\}
\end{aligned}
$$

Therefore, we can easily identify now:

1. the isolated elements, e.g., $B_{k, 1}, B_{k, 2}, B_{k, 3}, C_{k, 5}, C A_{k, 2}, C A_{k, 3}$, etc. and

2. the non-influential elements: $S_{k, 1}, S_{k, 6}, S_{k, 7}, S_{k, 8}$.

Notice that, in this particular example, we deal with a large number of variables, but unfortunately, we know only four simple dependence relations among them, which cannot permit better describing the real situation. It is 
clear that more dependencies exist (preferably composed and of superior levels), and a better description of the interaction (influence) between the involved elements can be obtained.

\section{Preliminaries and Notations in Hyperstructure Theory}

In the classical algebraic structures, the support sets are endowed with operations (called also compositions) that associate to a pair (or to an n-tuple) of elements only a single element of the considered set. The hypercompositional algebra deals with structures obtained as sets endowed with multi-valued operations, often called hyperoperations or hypercompositions. Thus, these structures are well known in the literature as hyperstructures, and their study started in 1934 with the definition and several properties of hypergroups presented by Marty [6] to the Eighth Congress of Scandinavian Mathematicians. More precisely, considering a non-empty set $H$ and denoting by $\mathcal{P}^{*}(H)$ the set of all non-empty subsets of $H$, a hyperoperation on $H$ is a mapping $\circ: H \times H \longrightarrow \mathcal{P}^{*}(H)$, and the structure $(H, o)$ is called a hypergroupoid. If we enlarge the codomain of the hyperoperation to the power set $\mathcal{P}(H)$, meaning that the image of some pairs of elements in $H$ could be the empty set, then $(H, \circ)$ is a partial hypergroupoid. If the hyperoperation satisfies the following axioms:

1. $(a \circ b) \circ c=a \circ(b \circ c)$ for all $a, b, c \in H$ (associativity),

2. $a \circ H=H \circ a=H$ for all $a \in H$ (reproductivity),

then the hypergroupoid $(H, \circ)$ is a hypergroup. Note that, for any non-empty subsets $A, B$ of $H$, there is $A \circ B=\bigcup\{a \circ b ; a \in A, b \in B\}$ and $A \circ a=A \circ\{a\}$ (and similarly, $a \circ A=\{a\} \circ A$ ) for any $a \in H$. A hypergroupoid satisfying the reproductivity is called a quasi-hypergroup, while a hypergroupoid satisfying the associativity is called a semihypergroup. If the weak-associativity holds, i.e., $a \circ(b \circ c) \cap(a \circ b) \circ c \neq \varnothing$ for all $a, b, c \in H$, then $(H, \circ)$ is called an $H_{v}$-group. Besides, the commutativity means that $a \circ b=b \circ a$ for any $a, b \in H$.

In the same foundational manuscript on hypergroups theory, Marty [6] also defined two induced hyperoperations (called the right and left division):

$$
\frac{a}{\mid b}=\{x \in H \mid a \in x \circ b\} \text { and } \frac{a}{b \mid}=\{x \in H \mid a \in b \circ x\},
$$

used by Jantosciak [7] and, later on, by many others researchers, as $a / b$ and $b \backslash a$, respectively. It is clear that in a commutative hypergroup, the right and left divisions coincide, being both denoted by $a / b$. These induced hyperoperations have a crucial role in the transposition axiom, i.e., $a / b \cap c / d \neq \varnothing \Longrightarrow$ $a \circ d \cap b \circ c \neq \varnothing$ for all $a, b, c, d \in H$, introduced by Prenowitz [8] to define the join spaces, used in the study, from an algebraic point of view, of the descriptive, projective, and spherical geometries. Thus, a join space is a commutative hypergroup satisfying the transposition axiom, and it has applications also in graph theory, automata theory, formal languages, etc.

A hyperoperation is called extensive [9,10] (or closed [11]) if the result of the hypercomposition of two elements always contains both elements, i.e., $a, b \in a \circ b$ for any $a, b \in H$. This aspect has been recently re-considered and studied in detail by Massouros et al. [12]. Notice that the reproduction axiom is an important consequence of the extensive property, i.e., any extensive hypergroupoid is a quasi-hypergroup.

Let us recall now some important roles that the elements of a hypergroupoid can play.

An element $a$ in a hypergroupoid $(H, \circ)$ is called a scalar, if $|a \circ b|=|b \circ a|=1$ for any $b \in H$. An element $e \in H$ is called a partial identity of $H$ if it is a left identity (i.e., there exists $a \in H$ such that $a \in e \circ a$ ) or a right identity (i.e., there exists $b \in H$ such that $b \in b \circ e$ ). We denote by $I_{p}$ the set of all partial identities of $H$. An element $e \in H$ is called an identity if it is simultaneously a left and right identity in $H$. Besides, for a given element $a \in H$, a partial identity of $a$ is an element $u \in H$ such that $a \in a \circ u \cap u \circ a$. The element $u \in H$ is a partial scalar identity of $a$ whenever from $a \in a \circ u \cap u \circ a$ it follows that $a=a \circ u=u \circ a$. The term "partial" here must not be confused with the "left or right" (identity), but it must be connected with the fact that the element $u$ has a partial behavior of identity 
with respect to the element $a$. We denote by $I_{p}(a)$ the set of all partial identities of $a$ and by $I_{p s}(a)$ the set of all partial scalar identities of $a$, while by $S c(H)$, we denote the set of all scalars of $H$. It is obvious that $I_{p s}(a)=I_{p}(a) \cap S c(H)$ for all $a \in H$.

Let $(H, \circ)$ be a hypergroup endowed with at least an identity. An element $a^{\prime} \in H$ is called an inverse of $a \in H$ if there exists an identity $e \in H$ such that $e \in a \circ a^{\prime} \cap a^{\prime} \circ a$. A hypergroup is called regular if it has at least one identity and each element has at least one inverse.

The idea of idempotency, important in, e.g., making distinctions between semigroups and groups, is transferred to hyperstructures in the usual way of inclusion. Notice that some authors, such as Polat [13], used a more strict definition of idempotency where $a * a=\{a\}$. This is motivated by the need to study geometry-motivated join spaces.

Definition 2. An element a of a semihypergroup $(H, \circ)$ is called an idempotent if $a \in a \circ a$. The semihypergroup $(H, \circ)$ is called an idempotent semihypergroup if all its elements are idempotent. A nonempty subset $A$ of a semihypergroup $(H, \circ)$ is called an idempotent subset if $A \subseteq A \circ A$.

We conclude this short overview of hypergroup theory with some aspects regarding the cyclicity, one of the main notions of algebraic groups and hypergroups. A recently-published insightful study on this topic can be found in [14]. Here, we recall only the definition given by Vougiouklis [15] and explained in detail in [14]:

Definition 3. A hypergroup $(H, \circ)$ is called cyclic if, for some $h \in H$, there is:

$$
H=h^{1} \cup h^{2} \cup \ldots \cup h^{n} \cup \ldots,
$$

where $h^{1}=\{h\}$ and $h^{m}=\underbrace{h \circ \ldots \circ h}_{m}$. If there exists $n \in \mathbb{N}$ such that Label (9) is finite, we say that $H$ is a cyclic hypergroup with finite period; otherwise, $H$ is a cyclic hypergroup with infinite period. The element $h \in H$ in Label (9) is called the generator of $H$, and the smallest power $n$ for which Label (9) is valid is called period of $h$. If all generators of $H$ have the same period $n$, then $H$ is called cyclic with period $n$. If, for a given generator h, Label (9) is valid, but no such n exists (i.e., Label (9) cannot be finite), then H is called cyclic with infinite period. If we can, for some $h \in H$, write:

$$
H=h^{n},
$$

then the hypergroup $H$ is called single-power cyclic with a generator $h$. If Label (9) is valid and for all $n \in \mathbb{N}$ and, for a fixed $n_{0} \in \mathbb{N}, n \geq n_{0}$ there is:

$$
h^{1} \cup h^{2} \cup \ldots \cup h^{n-1} \subsetneq h^{n}
$$

then we say that $H$ is a single-power cyclic hypergroup with an infinite period for $h$.

\section{Hypergroupoids Associated with Abstract Dependencies}

Regarding impact and influence, we can ask about the meaning of the following combinations:

1. $t \in \operatorname{Infl}(u)$, where $u \in \operatorname{Imp}(x)$;

2. $t \in \operatorname{Imp}(u)$, where $u \in \operatorname{Infl}(x)$;

3. $t \in \operatorname{Imp}(u)$, where $u \in \operatorname{Imp}(x)$;

4. $\quad t \in \operatorname{Infl}(u)$, where $u \in \operatorname{Infl}(x)$;

For 1 in the list, we have that $t \in \operatorname{Infl}(u)$ is equivalent to $u \in \operatorname{Imp}(t)$, and since $u \in \operatorname{Imp}(x)$, we have that $u \in \operatorname{Imp}(t) \cap \operatorname{Imp}(x)$. For 2 in the list, we have that $t \in \operatorname{Imp}(u)$ is equivalent to $u \in \operatorname{Infl}(t)$, and since $u \in \operatorname{Infl}(x)$, we have that $u \in \operatorname{Infl}(t) \cap \operatorname{Infl}(x)$. 
Thus, 1 and 2 in the list give us motivations for introducing the following hyperoperations:

$$
\begin{gathered}
x *_{1} y=\operatorname{Imp}(x) \cap \operatorname{Imp}(y) \\
x *_{2} y=\operatorname{Infl}(x) \cap \operatorname{Infl}(y)
\end{gathered}
$$

In this way, we can write that:

1. $t \in \operatorname{Infl}(u)$, where $u \in \operatorname{Imp}(x) \Rightarrow u \in t *_{1} x$;

2. $t \in \operatorname{Imp}(u)$, where $u \in \operatorname{Infl}(x) \Rightarrow u \in t *_{2} x$.

Regarding 3 and 4 in the list, we can ask whether these imply-in the case of 3 in the list-that $t \in \operatorname{Imp}(x)$, or -in case of 4 in the list-that $t \in \operatorname{Infl}(x)$. For 3 in the list, we indeed have that $t \in \operatorname{Imp}(u)$, where $u \in \operatorname{Imp}(x) \Rightarrow t \in \operatorname{Imp}(x)$ because we have that there exist dependences such that $t \sim D(u, \ldots)$ and $u \sim D(x, \ldots)$, which means that $t \sim D(u, D(x, \ldots), \ldots)$, which is equivalent to the fact that $x \in \operatorname{Infl}(t)$, which is equivalent to $t \in \operatorname{Imp}(x)$. For 4 in the list, we have that this is always true because we have that there exist dependences $u \sim D(t, \ldots)$ and $x \sim D(u, \ldots)$, which means that $x \sim D(u \sim D(t, \ldots), \ldots)$. This means that $t \in \operatorname{Infl}(x)$, or rather $t \in \operatorname{Infl}_{i}(x)$, to be more precise.

We can also ask about the implication of the fact that $x \in \operatorname{Infl}(y)$ for the sets $\operatorname{Imp}(x)$ and $\operatorname{Imp}(y)$.

Lemma 1. For an arbitrary pair of $x, y \in H$, the following implication holds: $x \in \operatorname{Infl}(y) \Rightarrow$ $\operatorname{Imp}(y) \subseteq \operatorname{Imp}(x)$.

Proof. First, notice that $x \in \operatorname{Infl}(y)$ is equivalent to $y \in \operatorname{Imp}(x)$, which is equivalent to the fact that there exists a dependence $y \sim D(x, \ldots)$. Now, take an arbitrary $u \in \operatorname{Imp}(y)$, which is equivalent to the fact that there exists a dependence $u \sim D(y, \ldots)$. Combined, these two facts mean that $u \sim D(y \sim D(x, \ldots), \ldots)$, which means that $x \in \operatorname{Infl}(u)$. However, this is equivalent to $u \in \operatorname{Imp}(x)$. In other words, we get that $\operatorname{Imp}(y) \subseteq \operatorname{Imp}(x)$.

Remark 2. Based on the duality that links impact and influence (see Remark 1), the implication in the above lemma can be also written as: $x \in \operatorname{Imp}(y) \Rightarrow \operatorname{Infl}(y) \subseteq \operatorname{Infl}(x)$.

As a "complement" of the hyperoperations (12) and (13), we shall regard also the following pair of hyperoperations:

$$
\begin{aligned}
& x *_{3} y=\operatorname{Imp}(x) \cup \operatorname{Imp}(y) \\
& x *_{4} y=\operatorname{Infl}(x) \cup \operatorname{Infl}(y)
\end{aligned}
$$

Obviously, since intersections can be empty, hyperstructures $\left(H, *_{1}\right)$ and $\left(H, *_{2}\right)$ can be partial hypergroupoids. However, this is not true for hyperstructures $\left(H, *_{3}\right)$ and $\left(H, *_{4}\right)$.

Theorem 1. Hypergroupoids $\left(H, *_{3}\right)$ and $\left(H, *_{4}\right)$ are commutative, extensive $H_{v}$-groups.

Proof. Commutativity and extensivity are obvious. In order to prove weak associativity, we need to find an element that, for arbitrary $x, y, z \in H$, belongs to the intersection of $\left(x *_{1} y\right) *_{1} z$ and $x *_{1}\left(y *_{1} z\right)$, or $\left(x *_{2} y\right) *_{2} z$ and $x *_{2}\left(y *_{2} z\right)$, respectively. However, thanks to the extensivity of both hyperoperations, these elements in question are $x, y, z$ in any case. The validity of the reproductive law is again a straightforward consequence of extensivity.

Remark 3. Of course, in a general case, the cardinality of the intersection discussed in the proof of Theorem 1 is greater than just trivially three. Indeed, it is easy to show that it always contains $\operatorname{Imp}(x) \cup \operatorname{Imp}(y) \cup \operatorname{Imp}(z)$, or $\operatorname{Infl}(x) \cup \operatorname{Infl}(y) \cup \operatorname{Infl}(z)$, respectively. 
Concentrate now on the idempotence of the above two hyperoperations " $*_{3}$ " and " $*_{4}$ ". Obviously, we have, for $n>2$, that $\underbrace{x *_{3} \ldots *_{3} x}_{n}=\operatorname{Imp}(x)$. However, in the case of $n=2$, the resulting set depends on the nature of $x$.

Lemma 2. If we regard $x^{n}=\underbrace{x *_{3} \ldots *_{3} x}_{n}$, then for an arbitrary $x \in H$, we get the following:

$$
x^{n}=x^{2}=\operatorname{Imp}(x) \quad \begin{cases}=\{x\} & \text { for isolated or non-influential } x \\ \neq\{x\} & \text { for } x \text { being an influential element of some } y\end{cases}
$$

Proof. It is obvious.

Overall, we get the following:

Lemma 3. As regards hyperoperation " $*_{3}$ ", there exists $n \in \mathbb{N}, n>1$, such that $H=x^{n}$ if and only if all elements of $H$ depend on $x$.

Proof. The fact that $H=x^{n}$ for some $x \in H$ and some $n \in \mathbb{N}$ is equivalent to the fact that for this $x$, we have $\operatorname{Imp}(x)=H$. Since $\operatorname{Imp}(x) \subseteq H$, we have to test only that $H \subseteq \operatorname{Imp}(x)$. This is equivalent to the fact that, for all $y \in H$, there is $y \in \operatorname{Imp}(x)$. However, this means that $x \in \operatorname{Infl}(y)$ for all $y \in H$; in other words, the fact that all elements $y \in H$ depend on $x$.

Remark 4. In Lemma 2, we showed that when looking for the smallest exponent $n$ such that $H=x^{n}$, $n$ cannot exceed two. This property can be linked to the idea of cyclicity in (semi)hypergroups [14,16,17]. To be more precise, the $H_{v}$-group $\left(H, *_{3}\right)$ is single-power cyclic with period two. However, notice that Vougiouklis [15] defined the concept for hypergroups, not for $\mathrm{H}_{v}$-groups.

If we regard hyperoperation " $*_{4}$ " instead of " $*_{3}$ ", we obtain a result similar (yet not the same!) to Lemma 2. Its proof is again obvious.

Lemma 4. If we regard $x^{n}=\underbrace{x *_{4} \ldots *_{4} x}_{n}$, then for an arbitrary $x \in H$, we get the following:

$$
x^{n}=x^{2}=\operatorname{Infl}(x) \quad \begin{cases}=\{x\} & \text { for } \text { isolated } x \\ \neq\{x\} & \text { for non-isolated } x\end{cases}
$$

Based on the duality between impact and influence, we straightforwardly obtain the following:

Lemma 5. As regards hyperoperation " $*_{4}$ ", we have $\underbrace{x *_{4} \ldots *_{4} x}_{n}=x^{2}=H$ if and only if $x$ depends on all elements of $H$.

Based on the above Theorem 1, the following natural question arises: When do we have $x *_{3} y=$ $\{x, y\}$ for any $x, y \in H$ (and the like for " $*_{4}$ ")? The answer is: never, on a set of cardinality greater than two. Indeed, if there exists at least one dependence relation, e.g., $a_{i, j} \sim D\left(a_{k, l}\right)$, then $\left\{a_{i, j}, a_{k, l}\right\} \subseteq$ $\operatorname{Imp}\left(a_{k, l}\right)$, and therefore, $\left\{a_{i, j}, a_{k, l}, a_{m, n}\right\} \subseteq a_{k, l} *_{3} a_{m, n}$. Therefore, $x *_{3} y=\{x, y\}$, or $x *_{4} y=\{x, y\}$, only if no dependence relation is defined, meaning that all elements of $H$ are isolated. However, such a case is trivial and not interesting for our purposes. Recall that Massouros and Mittas [18, 19] called such hypergroups $B$-hypergroups, where $B$ stands for binary, while Chvalina $[9,20]$ called them minimal extensive hypergroups. They have been recently used also in the study of breakable semihypergroups [21]. 
The following example deals with the "* $*_{3}$ " hyperoperation defined by (14).

Example 2. Let $H=\left\{a_{1,1}, a_{1,2}, a_{2,2}, a_{2,3}, a_{3,1}, a_{3,2}, a_{3,4}, a_{3,5}\right\}$, where $a_{1,1} \sim D\left(a_{1,2}, a_{2,2}, a_{3,2}, a_{3,5}\right), a_{2,2} \sim$ $D\left(a_{2,3}, a_{3,1}\right)$ and $a_{3,2} \sim D\left(a_{1,2}\right)$, which means that $a_{1,1} \sim D\left(a_{1,2}, a_{2,2} \sim D\left(a_{2,3}, a_{3,1}\right), a_{3,2} \sim\right.$ $\left.D\left(a_{1,2}, a_{3,5}\right)\right)$. Thus, for this composed dependence of $a_{1,1}$, there is level $(D)=2$ and length $(D)=$ 6 and $\operatorname{Infl}\left(a_{1,1}\right)=\operatorname{Infl}_{d}\left(a_{1,1}\right) \cup \operatorname{Infl}_{i}\left(a_{1,1}\right)=\left\{a_{1,1}, a_{1,2}, a_{2,2}, a_{3,2}, a_{3,5}\right\} \cup\left\{a_{1,2}, a_{2,3}, a_{3,1}\right\}, \operatorname{Infl}\left(a_{2,2}\right)=$ $\operatorname{Infl}_{d}\left(a_{2,2}\right)=\left\{a_{2,2}, a_{2,3}, a_{3,1}\right\}, \operatorname{Infl}\left(a_{3,2}\right)=\operatorname{Infl}_{d}\left(a_{3,2}\right)=\left\{a_{3,2}, a_{1,2}\right\}$. Regarding impact sets, we have: $\operatorname{Imp}\left(a_{1,1}\right)=\left\{a_{1,1}\right\}, \operatorname{Imp}\left(a_{1,2}\right)=\left\{a_{1,1}, a_{1,2}, a_{3,2}\right\}, \operatorname{Imp}\left(a_{2,2}\right)=\left\{a_{1,1}, a_{2,2}\right\}, \operatorname{Imp}\left(a_{2,3}\right)=\left\{a_{2,2}, a_{1,1}, a_{2,3}\right\}$, $\operatorname{Imp}\left(a_{3,1}\right)=\left\{a_{2,2}, a_{1,1}, a_{3,1}\right\}, \operatorname{Imp}\left(a_{3,2}\right)=\left\{a_{1,1}, a_{3,2}\right\}, \operatorname{Imp}\left(a_{3,4}\right)=\left\{a_{3,4}\right\}, \operatorname{Imp}\left(a_{3,5}\right)=\left\{a_{1,1}, a_{3,5}\right\}$. Notice that $\operatorname{Imp}\left(a_{3,4}\right)=\operatorname{Infl}\left(a_{3,4}\right)=\left\{a_{3,4}\right\}$, i.e., $a_{3,4}$ is isolated, while $\operatorname{Imp}\left(a_{1,1}\right)=\left\{a_{1,1}\right\}$ and $\left|\operatorname{Infl}\left(a_{1,1}\right)\right|>$ 1, i.e., $a_{1,1}$ is a non-influential element. Besides, we get the following hypercomposition table (notice that the hyperoperation is commutative) represented in Table 2.

Table 2. The hypercomposition table of $(H, *)$.

\begin{tabular}{|c|c|c|c|c|c|c|c|c|}
\hline$*$ & $a_{1,1}$ & $a_{1,2}$ & $a_{2,2}$ & $a_{2,3}$ & $a_{3,1}$ & $a_{3,2}$ & $a_{3,4}$ & $a_{3,5}$ \\
\hline$a_{1,1}$ & $\left\{a_{1,1}\right\}$ & $\begin{array}{c}\left\{a_{1,1},\right. \\
\left.a_{1,2}, a_{3,2}\right\}\end{array}$ & $\left\{a_{1,1}, a_{2,2}\right\}$ & $\begin{array}{c}\left\{a_{1,1}\right. \\
\left.a_{2,2}, a_{2,3}\right\}\end{array}$ & $\begin{array}{c}\left\{a_{1,1}\right. \\
\left.a_{2,2}, a_{3,1}\right\}\end{array}$ & $\left\{a_{1,1}, a_{3,2}\right\}$ & $\left\{a_{1,1}, a_{3,4}\right\}$ & $\left\{a_{1,1}, a_{3,5}\right\}$ \\
\hline$a_{1,2}$ & & $\begin{array}{c}\left\{a_{1,1}\right. \\
\left.a_{1,2}, a_{3,2}\right\}\end{array}$ & $\begin{array}{l}\left\{a_{1,1}, a_{1,2}\right. \\
\left.a_{2,2}, a_{3,2}\right\}\end{array}$ & $\begin{array}{c}\left\{a_{1,1}, a_{1,2}\right. \\
a_{2,2}, a_{2,3} \\
\left.a_{3,2}\right\}\end{array}$ & $\begin{array}{c}\left\{a_{1,1}, a_{1,2}\right. \\
a_{2,2}, a_{3,2} \\
\left.a_{3,1}\right\}\end{array}$ & $\begin{array}{c}\left\{a_{1,1}\right. \\
\left.a_{1,2}, a_{3,2}\right\}\end{array}$ & $\begin{array}{l}\left\{a_{1,1}, a_{1,2}\right. \\
\left.a_{3,2}, a_{3,4}\right\}\end{array}$ & $\begin{array}{l}\left\{a_{1,1}, a_{1,2}\right. \\
\left.a_{3,2}, a_{3,5}\right\}\end{array}$ \\
\hline$a_{2,2}$ & & & $\left\{a_{1,1}, a_{2,2}\right\}$ & $\begin{array}{c}\left\{a_{1,1}\right. \\
\left.a_{2,2}, a_{2,3}\right\}\end{array}$ & $\begin{array}{c}\left\{a_{1,1}\right. \\
\left.a_{2,2}, a_{3,1}\right\}\end{array}$ & $\begin{array}{c}\left\{a_{1,1}\right. \\
\left.a_{2,2}, a_{3,2}\right\}\end{array}$ & $\begin{array}{c}\left\{a_{1,1}\right. \\
\left.a_{2,2}, a_{3,4}\right\}\end{array}$ & $\begin{array}{c}\left\{a_{1,1}\right. \\
\left.a_{2,2}, a_{3,5}\right\}\end{array}$ \\
\hline$a_{2,3}$ & & & & $\begin{array}{c}\left\{a_{1,1}\right. \\
\left.a_{2,2}, a_{2,3}\right\}\end{array}$ & $\begin{array}{l}\left\{a_{1,1}, a_{2,2}\right. \\
\left.a_{2,3}, a_{3,1}\right\}\end{array}$ & $\begin{array}{l}\left\{a_{1,1}, a_{2,2}\right. \\
\left.a_{2,3}, a_{3,2}\right\}\end{array}$ & $\begin{array}{l}\left\{a_{1,1}, a_{2,2}\right. \\
\left.a_{3,4}, a_{2,3}\right\}\end{array}$ & $\begin{array}{l}\left\{a_{1,1}, a_{2,2}\right. \\
\left.a_{2,3}, a_{3,5}\right\}\end{array}$ \\
\hline$a_{3,1}$ & & & & & $\begin{array}{c}\left\{a_{1,1}\right. \\
\left.a_{2,2}, a_{3,1}\right\}\end{array}$ & $\begin{array}{l}\left\{a_{1,1}, a_{2,2}\right. \\
\left.a_{3,1}, a_{3,2}\right\}\end{array}$ & $\begin{array}{l}\left\{a_{1,1}, a_{2,2}\right. \\
\left.a_{3,1}, a_{3,4}\right\}\end{array}$ & $\begin{array}{l}\left\{a_{1,1}, a_{2,2}\right. \\
\left.a_{3,1}, a_{3,5}\right\}\end{array}$ \\
\hline$a_{3,2}$ & & & & & & $\left\{a_{1,1}, a_{3,2}\right\}$ & $\begin{array}{c}\left\{a_{1,1}\right. \\
\left.a_{3,2}, a_{3,4}\right\}\end{array}$ & $\begin{array}{c}\left\{a_{1,1},\right. \\
\left.a_{3,2}, a_{3,5}\right\}\end{array}$ \\
\hline$a_{3,4}$ & & & & & & & $\left\{a_{3,4}\right\}$ & $\begin{array}{c}\left\{a_{1,1},\right. \\
\left.a_{3,4}, a_{3,5}\right\}\end{array}$ \\
\hline$a_{3,5}$ & & & & & & & & $\left\{a_{1,1}, a_{3,5}\right\}$ \\
\hline
\end{tabular}

According to Theorem $1,\left(H, *_{3}\right)$ is a commutative, extensive $H_{v}$-group. It is obvious that all elements of $H$ are hyperstructure identities of $H$, and no element is a scalar identity. Moreover, any influential element of a given element $x \in H$ is a partial identity of $x$, i.e., $I_{p}(x)=\operatorname{Infl}(x)$. Furthermore, thanks to the extensivity and the fact that every element of $H$ is a hyperstructure identity, we have that for any $x \in H$, the set of inverses of $x$, usually denoted $i(x)$, equals $H$.

Let us now focus back on hyperoperations " $*_{1}$ " and " $*_{2}$ ". We have already mentioned that in the case of these hyperoperations, we can arrive at partial hypergroupoids. Let us therefore ask when this can happen.

Lemma 6. If $H$ contains at least one isolated element, or at least one non-influential element $x$ such that $\operatorname{Infl}(x) \neq H$, then $\left(H, *_{1}\right)$ is a partial hypergroupoid.

Proof. Let us start with the first hyperoperation. We need to find at least two elements $x, y \in H$ such that $x *_{1} y=\varnothing$. It is obvious that we must regard $x \neq y$. If $x$ is isolated, then $\operatorname{Imp}(x)=\{x\}$, but at the same time $x \notin \operatorname{Imp}(y)$. Therefore, $\operatorname{Imp}(x) \cap \operatorname{Imp}(y)=\varnothing$. If $x$ is non-influential and $\operatorname{Infl}(x) \neq H$, 
then $\operatorname{Imp}(x)=\{x\}$, and there exists $y \in H, y \neq x$ such that $y \notin \operatorname{Infl}(x)$, equivalently with $x \notin \operatorname{Imp}(y)$, thereby $x *_{1} y=\varnothing$.

Lemma 7. If $H$ contains at least one isolated element, then $\left(H, *_{2}\right)$ is a partial hypergroupoid.

Proof. This is similar to the proof for the hyperoperation " $*_{1}$ ".

Remark 5. Notice that if $H$ contains at least one non-influential element, the answer regarding partial hypergroupoid $\left(H, *_{2}\right)$ is not the same as for hyperoperation " $*_{1}$ ". Indeed, $\operatorname{Infl}(x) \cap \operatorname{Infl}(y)=\varnothing$ is equivalent to the fact that there does not exist an element $z \in H$ such that both $x$ and $y$ depend on $z$, i.e., in the notation of dependencies, $x \sim D(z, \ldots)$ and simultaneously $y \sim D(z, \ldots)$. Intuitively, this can be linked tot the notion of "separability", inner irreducibility [22], or complete hypergroups (e.g., see [23]).

Now, we shall discuss the issue of the extensivity of hyperoperations " $*_{1}$ " and " $*_{2}$ ". Suppose that the hyperoperation " $*_{1}$ " is extensive. This means that, for all $x, y \in H$, there is $\{x, y\} \subseteq \operatorname{Imp}(x) \cap$ $\operatorname{Imp}(y)$. Since, for all $x \in H$, there is $x \in \operatorname{Imp}(x)$, the above relation means that $x \in \operatorname{Imp}(y)$ and $y \in \operatorname{Imp}(x)$. However, given Lemma 1, this means that $\operatorname{Infl}(x)=\operatorname{Infl}(y)$, and that for all $x, y \in H$. However, $x \in \operatorname{Imp}(y)$ is equivalent to $y \in \operatorname{Infl}(x)$, and $y \in \operatorname{Imp}(x)$ is equivalent to $x \in \operatorname{Infl}(y)$. Therefore, we get also that $\operatorname{Imp}(x)=\operatorname{Imp}(y)$, for all $x, y \in H$. Moreover, from the conjunction $y \in \operatorname{Infl}(x)$ and $x \in \operatorname{Infl}(y)$, we get that $\{x, y\} \subseteq \operatorname{Infl}(x) \cap \operatorname{Infl}(y)$, i.e., the hyperoperation " $*_{2}$ " is extensive.

Now, what are the implications of the simultaneous validity of $\operatorname{Imp}(x)=\operatorname{Imp}(y)$ and $\operatorname{Infl}(x)=$ $\operatorname{Infl}(y)$ for all $x, y \in H$ ? This means that $\operatorname{Imp}(x)=\operatorname{Infl}(x)=H$, for all $x \in H$. Thus, for example, if $H=x_{1}, \ldots, x_{n}$, then we have $x_{1} \sim D\left(x_{1}, \ldots x_{n}\right), \ldots, x_{n} \sim D\left(x_{1}, \ldots x_{n}\right)$, which means that each element of $H$ depends on all elements in the set, which is the extreme case opposite the case when all elements are isolated. Since in real-life contexts, one cannot assume such an extreme case, we will not assume the extensivity of hyperoperations " $*_{1}$ " and " $*_{2}$ " based on the intersections of influential or impact sets.

Finally, let us mention the idempotence of hyperoperations " $*_{1}$ " and " $*_{2}$ ". Since $\operatorname{Imp}(x) \cap \operatorname{Imp}(x)=$ $\operatorname{Imp}(x)$, it is obvious that $\underbrace{x *_{1} \ldots *_{1} x}_{n}=x^{n}=x^{2}=\operatorname{Imp}(x)$ for all $x \in H$ and $n>2$ (and the same for " $*_{2}$ "). Thus, we arrive at the same conclusions as for hyperoperations " $*_{3}$ " and " $*_{4}$ " in Lemmas 2 and 4 .

\section{Conclusions and Future Work}

The links between binary (or $n$-ary) relations or fuzzy relations and algebraic hyperstructures represent currently an important line of research, developed both from a theoretical point of view [24-27] and also for the applications in social science [28], code theory [29], graph theory [30], automata theory [22,31], etc. In this article, motivated by the special interdependencies existing between the variables used for the weather forecasting, we introduced a new type of relation, called dependence relations, and started the study of their algebraic properties. We associated with them four hyperoperations and constructed the corresponding hyperstructures, having in mind providing a new interpretation of these dependencies by using the elements of the theory of algebraic hyperstructures, i.e., idempotence, cyclicity, extensivity.

Notice that, in our context, some properties of the dependencies such as length or the cardinality of the sets $\operatorname{Imp}(x)$ or $\operatorname{Infl}(x)$ can be better described by numerical values, which can be then compared or ordered using the standard ordering of natural/real numbers. Moreover, this comparison can be applied not only to the given dependencies, but also to their pairs. For example, we can look for all dependencies whose length exceeds the maximum of lengths of two given dependencies, or all dependencies such that the cardinality of their impact sets is greater than the minimum of the cardinalities of the impact sets of two given dependencies, etc. This technique is similar to 
the one employed, e.g., in Chvalina [20], Chapter 6 or Massouros and Mittas [18] in the theory of automata, where lengths of compound words were studied from this perspective. Notice also that Al Tahan and Davvaz [32] used this technique to construct cyclic hypergroups of an arbitrary braid group of $n$ brands. In this respect, we may in fact directly apply many results regarding EL-hyperstructures $[10,14,33]$. Besides, the aspect regarding the influence of one element on the others can numerically be characterized by a parameter, called the degree of influence of an element with respect to another element, which can be interpreted easily as "the higher degree the stronger dependence", because the dependence on direct influential elements is stronger, i.e., more significant, than the dependence on indirect influential elements. These are only some initial proposals to describe numerically the relations among the elements within this kind of dependencies. In our future work, we intend to better define them and also connect them with fuzzy set theory and graph theory. Regarding the second one, we may associate a directed graph with arcs $(x, y)$ whenever $y \in \operatorname{Imp}(x)$, that is a reflexive transitive graph, with $\operatorname{Imp}(x)$ the out-neighborhood of $x$ and $\operatorname{Infl}(x)$ its in-neighborhood.

Author Contributions: Conceptualization, I.C., J.K., and M.N.; investigation, I.C., J.K., and M.N.; methodology, I.C., J.K., and M.N.; writing, original draft, I.C., J.K., and M.N.; writing, review and editing, I.C., J.K., and M.N.

Funding: The first and the second author acknowledge the project "Method for the forecasting of local radiological pollution of atmosphere using Gaussian process models", ID L2-8174, which was financially supported by the Slovenian Research Agency. The second author acknowledges also research core funding No. P2-0001, which was financially supported by the Slovenian Research Agency. The third author is supported by the FEKT-S-17-4225 grant of Brno University of Technology.

Conflicts of Interest: The authors declare no conflict of interest.

\section{References}

1. Armstrong, W.W. Dependency structures of database relationships. In Proceedings of IFIP Congress, Stockholm, Sweden, 5-10 August 1974; 580-583.

2. Matúš, F. Abstract functional dependency structures. Theor. Comput. Sci. 1991, 81, 117-126. [CrossRef]

3. More, S.M.; Naumov, P. The Functional Dependence Relation on Hypergraphs of Secrets. In Computational Logic in Multi-Agent Systems. CLIMA 2011. Lecture Notes in Computer Science; Leite, J., Torroni, P., Agotnes, T., Boella, G., Van der Torre, L., Eds.; Springer: Berlin/Heidelberg, Germany, 2011; Volume 6814.

4. Jančič, M.; Kocijan, J.; Grašič, B. Identification of atmospheric variable using Deep Gaussian processes. IFAC-PapersOnLine 2018, 51, 43-48. [CrossRef]

5. Kocijan, J.; Perne, M.; Mlakar, P.; Grašič, B.; Zlata Božnar, M. Hybrid model of the near ground temperature profile. Stoch. Environ. Res. Risk. Assess. 2019, submitted.

6. Marty, F. Sur une généralisation de la notion de groupe. In Proceedings of the VIII Congrès des Mathématiciens Scandinaves, Stockholm, Sweden, 1934; pp. 45-49.

7. Jantosciak, J. Transposition hypergroups, Noncommutative Join Spaces. J. Algebra 1997, 187, $97-119$. [CrossRef]

8. Prenowitz, W. A contemporary approach to Classical Geometry. Am. Math. Mon. 1961, 68 Pt 2, 1-67. [CrossRef]

9. Chvalina, J. Commutative hypergroups in the sense of Marty and ordered sets. In Proceedings of the Summer School on General Algebra and Ordered Sets, Olomouc, Czech Republic, 4-12 September 1994; pp. 19-30.

10. Novák, M.; Křehlík, Š. EL-hyperstructures revisited. Soft Comput. 2018, 22, 7269-7280. [CrossRef]

11. Massouros, C.G. On path hypercompositions in graphs and automata. MATEC Web Conf. 2016, 41, 05003. [CrossRef]

12. Massouros, C.G.; Massouros, G.G. On open and closed hypercompositions. In AIP Conference Proceedings 1978, Proceedings of the International Conference on Numerical Analysis and Applied Mathematics (ICNAAM 2017), The MET Hotel, Thessaloniki, Greece, 25-30 September 2017; American Institute of Physics Publishing: Melville, NY, USA, 2018.

13. Polat, N. On bipartite graphs whose interval space is a closed join space. J. Geom. 2017, 108, 719-741. [CrossRef] 
14. Novák, M.; Křehlík, Š.; Cristea, I. Cyclicity in EL-hypergroups. Symmetry 2018, 10, 611. [CrossRef]

15. Vougiouklis, T. Cyclicity in a special class of hypergroups. Acta Univ. Carolinae Math. Phys. 1981, 22, 3-6.

16. Al Tahan, M.; Davvaz, B. On some properties of single power cyclic hypergroups and regular relations. J. Algebra Appl. 2017, 16, 1750214. [CrossRef]

17. De Salvo, M.; Freni, D. Semi-ipergruppi e ipergruppi ciclici. Atti Sem. Mat. Fis. Univ. Modena 1981, 30, 44-59.

18. Massouros, G.G.; Mittas, J.D. Languages, Automata and Hypercompositional Structures. In Proceedings of the Fourth International Congress on Algebraic Hyperstructures and Applications, Xanthi, Greece, 27-30 June 1990; World Scientific Publishing Co. Pte. Ltd.: Singapore, 1991; pp. 137-147.

19. Massouros, G.G. Hypercompositional structures from the computer theory. Ratio Math. 1999, 13, 37-42.

20. Chvalina, J. Functional Graphs, Quasi-ordered Sets and Commutative Hypergroups; Masaryk University: Brno, Czech Republic, 1995. (In Czech)

21. Heidari, D.; Cristea, I. Breakable semihypergroups. Symmetry 2019, 11, 100. [CrossRef]

22. Chvalina, J.; Chvalinová, L. State hypergroups of automata. Acta Math. Inform. Univ. Ostrav. 1996, 4, $105-120$.

23. Cristea, I.; Hassani Sadrabadi, E.; Davvaz, B. A fuzzy application of the group $\mathbb{Z}_{n}$ to complete hypergroups. Soft Comput. 2019. [CrossRef]

24. Cristea, I. Regularity of intuitionistic fuzzy relations on hypergroupoids. An. Şt. Univ. Ovidius Constanţa 2014, 22, 105-119. [CrossRef]

25. Cristea, I.; Ştefănescu, M. Hypergroups and n-ary relations. Eur. J. Combin. 2010, 31, 780-789. [CrossRef]

26. De Salvo, M.; Lo Faro, G. Hypergroups and binary relations. Multi. Val. Log. 2002, 8, 645-657.

27. Leoreanu-Fotea, V.; Davvaz, B. n-hypergroups and binary relations. Eur. J. Combin. 2008, 29, 1207-1218. [CrossRef]

28. Hošková-Mayerová, Š.; Maturo, A. Decision-making process using hyperstructures and fuzzy structures in social sciences. Stud. Fuzz. Soft Comput. 2018, 357, 103-111.

29. Saeid, A.B.; Flaut, C.; Hoškova-Mayerova, Š.; Afshar, M.; Rafsanjani, M.K. Some connections between BCK-algebras and n-ary block codes. Soft Comput. 2018, 22, 41-46. [CrossRef]

30. Kalampakas, A.; Spartalis, S.; Tsigkas, A. The Path Hyperoperation. An. Şt. Univ. Ovidius Constanţa 2014, 22, 141-153. [CrossRef]

31. Chvalina, J.; Hošková-Mayerová, Š.; Nezhad, A.D. General actions of hyperstructures and some applications. An. Şt. Univ. Ovidius Constanţa 2013, 21, 59-82. [CrossRef]

32. Al Tahan, M.; Davvaz, B. On a special single-power cyclic hypergroup and its automorphisms. Discret. Math. Algorithm. Appl. 2016, 8, 1650059. [CrossRef]

33. Novák, M.; Cristea, I. Composition in EL-hyperstructures. Hacet. J. Math. Stat. 2019, 48, 45-58. [CrossRef] 